\title{
Familiar Music Listening with Binaural Beats for Older People with Depressive Symptoms in Retirement Homes
}

\author{
Huei-Chuan Sung ${ }^{1,4, \dagger}$, Wen-Li Lee ${ }^{2}$, Hao-Ming Li ${ }^{3}$, Chia-Ying Lin ${ }^{4}$, Yu-Zu Wu ${ }^{5}$, Jing-Jy Wang ${ }^{6}$, Tzai-Li Li ${ }^{7}$
}

\begin{abstract}
Objective

Depression affect not only older people' self-care ability and quality of life, but also increase the burden of their families and caregivers as well as health care resources. With consideration of the adverse effects of antidepressant medications and high medical costs of treating depression, it is important to use non-pharmacological interventions for managing depressive symptoms of older people. This study aimed to evaluate the effects of familiar music with alpha binaural beats on blood pressure, heart rate variability, and depression level of older people with depressive symptoms and to assess its feasibility for older people.
\end{abstract}

\section{Methods}

This study used a one-group pre-test and post-test design. Thirty-five older people residing in a retirement home participated in the study. Each participant listened to 30-minute familiar music with alpha binaural beats via a headphone individually while lying on the chair or bed in the afternoon in a private room. The music compact disk were Taiwanese old songs from the 1950-1970s which was familiar to most of the Taiwanese older people.

\section{Results}

The study results indicated that the participants after listening to 30-minute familiar music with embedded binaural beats of alpha frequencies at $10 \mathrm{~Hz}$ had shown immediate effects by a significant reduction on their heart rate, systolic blood pressure, and LF norm of heart rate variability (HRV), and a significant increase on HF norm of HRV indicating that the participants were in a relaxing state after listening to the music. Depression level was significantly decreased after listening to 30-minute familiar music with alpha binaural beats in the afternoon daily for five days.

\section{Conclusions}

Familiar music listening with alpha binaural beats can be a noninvasive and suitable intervention to promote relaxation and alleviate negative emotion in older people with depressive symptoms in long-term care settings.

\section{Keywords}

Depression; Depressive symptom; Music; Binaural beat; Older adult; Elder

\section{Introduction}

Depression is prevalent among older people. The prevalence of depression is $10 \%$ to $25 \%$ in community-dwelling older people [1] and up to $48 \%$ to $50 \%$ in nursing homes and medical settings [2]. In Taiwan, around $13-21 \%$ older people diagnosed with depression [3] and $52.05 \%$ of institutionalized elderly residents had depression [4]. Depression has a large impact in older people and been found to be a significant risk factor contributing to death, particularly

'Graduate Institute of Long-term Care, Department of Nursing, Tzu Chi University of Science and Technology, Hualien, Taiwan

${ }^{2}$ Department of Medical Imaging and Radiological Sciences, Tzu Chi University of Science, Hualien, Taiwan

${ }^{3}$ Department of Psychiatry, Buddhist Tzu Chi General Hospital, Hualien, Taiwan

${ }^{4}$ Taiwanese Center for Evidence-based Health Care, Hualien, Taiwan

${ }^{5}$ Department of Physical Therapy, Tzu Chi University, Hualien, Taiwan

${ }^{6}$ Department of Nursing, College of Medicine, National Cheng Kung University, Tainan, Taiwan

${ }^{7}$ Department of Sport Promotion, National Taiwan Sport University, Taoyuan, Taiwan

${ }^{\dagger}$ Author for correspondence: Huei-Chuan Sung, RN, PhD, Associate professor, Institute of long-term care \& Department of Nursing, Tzu Chi University of Science and Technology, \& Taiwanese center for Evidence-based Health Care, No.880, Sec. 2, Chien-Kuo Rd., Hualien 970, Taiwan. Tel: 886-3-8572158 ext.2435, Fax: 886-3-8577962, email: sung@tcust.edu.tw 
those with poor physical health. Older people with depression commit suicide more than other age group and in those who survive, the prognosis is worse in older people [5]. Unfortunately, depression remains underdiagnosed and inadequately treated in older people in the clinical settings [6], and older people are especially unlikely to seek professional mental health services [7]. Untreated depression affect not only older people' self-care ability and quality of life, but also increase the burden of their families and caregivers as well as health car resources [8].

Older people diagnosed with depression are traditionally treated with antidepressants. The adverse consequences of antidepressants may be more common in the treatment of depression in older population than in younger age groups owing to higher levels of comorbidity, physical and psychological changes and polypharmacy [9]. The adverse effects associated with antidepressants in older people with depression include attempted suicide and self harm, all-case mortality and stroke, rate of falls and fractures, and epilepsy and seizures [9]. In addition, the modest increase in antipsychotic prevalence in the older population has been associated with a substantial increase in cost [10]. With consideration of the adverse effects and high costs of antidepressants used in the elderly population, it is important to develop inexpensive and viable non-pharmacological interventions to manage depression in older people. There are several nonpharmacological approaches suggested for treating depression in older people, such as relaxation techniques, biofeedback [11], cognitive and behavioural therapy $[12,13]$, therapeutic recreation, music therapy $[14,15]$, and exercise [16].

Music has been shown to positively influence a broad range of outcomes including alleviating anxiety, promoting relaxation, improving mood, reducing pain, decreasing agitation, improving exercise performance and increasing food intake among various populations [17]. Music can facilitate feelings of physical and mental relaxation by masking environmental noises and refocusing an individual's attention on a more pleasant emotional state [18]. It is believed that music as an auditory stimulation can occupy some of the neurotransmitters, therefore affect the autonomic nervous system and divert feelings of anxiety [19]. Several neuroscience studies also found that listening to preferred music can induce pleasant and positive feelings, and the pleasurable feeling was correlated with activation of the limbic system $[20,21]$.
In the last decade, binaural beats technology has gained considerable focus and has been suggested to have positive impact on mood problems. Music products in which binaural beats are embedded in music have also been created to facilitate mood effects. Binaural beats have been used to entrain the brain toward a desired frequency. When two tones of a slightly different frequency are presented separately through headphones to each auditory channel, the listener perceives a third tone, which is known as the binaural beat [22,23]. Inducing brain-wave states with binaural auditory beats has been used to decrease anxiety and behavioral problems, and improve mood [24].

Several studies found that music with binaural beats can induce relaxation, and therefore anxiety can be reduced [24-26]. Few studies examined mood with auditory stimulation using binaural beats of delta or beta in healthy adults, but found no effect with depression [23,27]. A recent review suggested that alpha stimulation was preferable for relaxation, short-term stress, and pain relief, but the effectiveness of binaural beats for mood need further research [28].

Binaural beats are often embedded in music. The music used in this special binaural beats technology may play an important role in its effect. Studies suggested that music preferences may be an important factor for the effect of music on physiological and psychological outcomes [29]. Previous studies related to use of binaural beats technology mainly focus on healthy adults or patients prior to day surgery, but very limited studies focus on effect of binaural beats on older people. The majority of previous studies used western music with embedded binaural beats and had some methodological limitations. Limited evidence is available about the effect of familiar music with alpha binaural beats on depressive symptoms of older people.

\section{Methods}

This study used a one-group pre-test and posttest design and aimed to evaluate the effects of familiar music with alpha binaural beats on blood pressure, heart rate variability, and depression level of older people with depressive symptoms and to assess its feasibility for older people.

\section{- Participants}

The participants were recruited from a large residential care facility in Taiwan. Inclusion criteria were: age of 65 or older, short version of Geriatric Depression Scale (GDS-15) greater 
than five, cognitive intact, no severe hearing impairment, and no obvious symptoms of acute pain or infection. A convenience sample of 38 participants who met the inclusion criteria were invited and consented to participate in the study. Thirty-five participants completed the study with two withdrew because of physical discomfort and one relocation.

\section{- Intervention}

An auditory compact disk consisted of recordings of 30-minute alpha binaural beats with an overlay of familiar music produced by a Taiwanese music composer. Prior to the intervention, the participants were surveyed with their preferences and familiarity of music. The music used in this compact disk were Taiwanese old songs from the 1950-1970s which was familiar to most of the Taiwanese older people and to this group of participants. The auditory stimuli of alpha binaural beats were generated with Mind Station software. The purpose of application of binaural beats is to entrain brain wave activity. When an individual listens to binaural beats at $10 \mathrm{~Hz}$, his/ her brain would produce a $10-\mathrm{Hz}$ beat, which is corresponding to an alpha pattern of brain wave activity associated with relaxation [22]. In this study, binaural beats at $10 \mathrm{~Hz}$ was used. A binaural beat frequency of $10 \mathrm{~Hz}$, which came from source frequencies of $350 \mathrm{~Hz}$ left and 360 $\mathrm{Hz}$ right, was embedded into the recordings of the 30-minute familiar music.

\section{- Measures}

Blood pressure was measured by a digital blood pressure monitor (Omron Healthcare, USA). Systolic and diastolic blood pressures were used for data analysis.

HRV was measured using the NeXus-10 wireless device with Biotrace+ Software (Mind Media B.V. Herten, Netherlands). The NeXus-10 Device recorded heart rate using a standard 3-lead ECG at $1024 \mathrm{~Hz}$. The Nexus-10 software allowed for analysis of heart rate data and provided HRV parameters. High values of the low-to-high (LF/HF) ratio indicate a dominance of sympathetic activity, while low values indicate a dominance of parasympathetic activity. When experiencing anxiety, the sympathetic nerves will be activated, and can cause the heart rate to increase, high frequency (HF) to decrease, and low frequency (LF) and the LF/HF ratio to increase. LF/HF ratio is a widely used HRV index of sympathovagal balance between the sympathetic and parasympathetic branches, and their interactions of the autonomic nervous system [30].

Depression level was measured by the short version of Geriatric Depression Scale (GDS-15) [31]. The GDS-15 is a very common measure used to assess depression level in the elderly population in the clinical settings. A summary of all 15 questions yields a total score from 0-15, with a score greater than five indicating being depressive. GDS-15 is a valid and reliable tool with a Cronbach's alpha coefficient of 0.94 [32].

\section{- Procedures}

The study was conducted in a large long-term care facility in Taiwan. After obtaining approval from the institutional review board (IRB) and the facility administrators, participants who met the inclusion criteria were approached and invited to participate. The researcher explained the study proposal to the participants individually. Only participants who provided informed consent participated in the study and they were reassured of confidentiality and anonymity. They were also informed that participation in this study was voluntary and no loss of benefits to which they entitled would occur if they decided not to participate.

Baseline assessment was conducted for all participants, including demographics, GDS-15, blood pressure using a digital blood pressure monitor and heart rate variability using NeXus-10 wireless device prior to the music listening intervention. The music listening intervention was implemented between 2:30 4:00 pm in the afternoon. Each participant was asked to be seated comfortably in a reclined chair in a dimly lit quite private room or lying on the bed in their own room. Participants were asked to rest comfortably with eyes closed. The participant used headphones and rested quietly with no audio stimulation for five minutes, and then listened to 30 minutes of familiar music with alpha binaural beats played via a CD player, and then rested for five minutes with no audio stimulation. Each participant's blood pressure was measured before and after the music listening session. Their heart rate variability were measured using NeXus-10 wireless biofeedback for 40 minutes, including five minutes with no audio stimulation at pretest, 30 minutes during the music listening, and five minutes with no audio stimulation at posttest. Depression level was assessed again as posttest using GDS-15 after the participants listened to the 30-minute familiar music with binaural beats in the afternoon daily for five days. 


\begin{abstract}
Data analysis
Data were managed and analyzed using the Statistical Package for the Social Sciences (SPSS) version 20.0 (SPSS Inc., Chicago, IL, USA). Descriptive statistics were used to summarize the characteristics of the sample. Due to the small sample size, non-parametric statistics was used. Wilcoxon Signed Rank test was used to examine differences in pre-test and post-test heart rate, systolic blood pressure, diastolic blood pressure, low frequency (LF norm) of HRV, high frequency (HF norm) of HRV, and LF/HF ratio of HRV, and depression level. Statistical significance was set at the conventional $\mathrm{p}<0.05$ level (two-tailed).
\end{abstract}

\section{Results}

Thirty-five older people completed the study. The mean age for this sample was 78.86 (SD 7.37), ranging from 67-97 years. The majority of the participants were male (74.3\%). Close to half of the participants had received no formal education (48.6\%). The average numbers of medical diagnoses for the participant was 2.8 (SD 1.16) and average number of medication taken was 1.85 (SD 1.09). Only $20 \%$ of the participants were mobile without assistance.

\section{- Immediate effect of familiar music with binaural beats on blood pressure and HRV}

The participants' systolic blood pressure was reduced from a mean of $128.57 \mathrm{mmHg}$ (SD $16.94)$ at pre-test to $119.97 \mathrm{mmHg}(\mathrm{SD} 14.79)$ at post-test after listening to 30-minute music. Wilcoxon signed rank test result reveal that the participants' mean systolic blood pressure was significantly decreased after listening to the 30-minute familiar music with alpha binaural beats $(Z=-5.117, p<0.001)$ (Table 1$)$.

The participants' diastolic blood pressure was reduced from $71.91 \mathrm{mmHg}(\mathrm{SD} 13.81)$ at pretest to $71.51 \mathrm{mmHg}$ (SD 12.13) at post-test. Wilcoxon signed rank test result indicated that the reduction of the participants' mean diastolic blood pressure after listening to the 30-minute familiar music with alpha binaural beats did not reach significant difference $(Z=-0.349, p=0.73)$ (Table 1).

After listening to the 30-minute familiar music with alpha binaural beats, the participants' mean heart rate was reduced from 70.11 (SD 9.3) at pre-test to 66.69 (SD 9.48) at post-test. Wilcoxon signed rank test result indicated that the participants' mean heart rate was significantly decreased after listening to the familiar music with alpha binaural beats $(Z=-4.075, p<0.001)$ (Table 1).

The LF norm was decreased from a mean of 37.27 (SD 11.73) at pre-test to 31.51 (SD 13.57) at post-test. Wilcoxon signed rank test result indicated that the decrease of the participants' LF norm after listening to the familiar music with alpha binaural beats reached a significant difference $(Z=-2.342, p=0.019)$. The HF norm was increased from a mean of 45.81 (SD 14.86) at pre-test to 50.5 (SD 18.01) at post-test. Wilcoxon signed rank test result showed that the increase of the participants' HF norm after listening to the familiar music with alpha binaural beats reached a significant difference indicating the participants were in a state of relaxation $(Z=-$ $1.974, p=0.048)$. The participants' LF/HF ratio was decreased from a mean of 0.92 (SD 0.70) at pre-test to 0.80 (SD 0.51) at post-test. Wilcoxon signed rank test result showed that the decrease of the participants' LF/HF ratio after listening to the familiar music with alpha binaural beats did not reach significantly difference $(Z=-1.029$, $p=0.304$ ) (Table 1).

\section{- Five-day effect of familiar music with binaural beats on depression}

After listening to the familiar music with alpha binaural beats 30 minutes in the afternoon for five days, the mean depression score was significantly decreased from 10.97 (SD 1.51) at pre-test to 7.61 (SD 2.08) at post-test. Wilcoxon signed rank test result indicated that the participants' mean depression score was significantly decreased after listening to the familiar music with alpha binaural beats after five days $(Z=-4.83, p<0.001)$ (Table 1).

\section{Discussion}

This study evaluated the effects of familiar music with alpha binaural beats on blood pressure, heart rate variability, and depression of older people with depressive symptoms. The study results indicated that the participants after listening to 30-minute familiar music with embedded binaural beats of alpha frequencies at $10 \mathrm{~Hz}$ had shown immediate effects by a significant reduction on their heart rate, systolic blood pressure, and LF norm of HRV, and a significant increase on HF norm of HRV indicating that the participants were in a relaxing state after listening to the music. In our study, the participants had a significant reduced LF norm and a significant increased HF norm in their heart rate variability 
after 30-minute music listening, which indicated that the parasympathetic nervous system was activated and in a relaxing state. In our study, the immediate effects on blood pressure and heart rate variability were shown to benefit from a single session of familiar music listening with alpha binaural beats. Literature suggests that repeated exposure to binaural beats may allow the listener to enter the desired brain states and show more significant positive effects [28].

The purpose of application of binaural beats is to entrain brain wave activity. The reason we chose to use binaural beats of alpha frequencies (10 $\mathrm{Hz}$ ) was based on Oster's suggestions [22]. $\mathrm{He}$ suggested that binaural beats at $10 \mathrm{~Hz}$ produce a $10-\mathrm{Hz}$ beat, which is corresponding to an alpha pattern of brain wave activity associated with relaxation. Alpha brainwave frequencies (8-12 $\mathrm{Hz}$ ) can reflect a calm and peaceful state.

Our study results showed that the depression level was significantly decreased after listening to 30-minute familiar music with alpha binaural beats in the afternoon daily for five days. We also found that there was a negative correlation between relaxation and depression. The higher the relaxation level was, the lower the depression level after listening to the music intervention. This may be explained that the participants felt less depressed when they were more relaxed after listening to the familiar music with alpha binaural bests which is associated with relaxation [22].

Our study was the first study which evaluated the effect of familiar music with binaural beats on older people with depressive symptoms. The majority of previous studies evaluating the effect of binaural beats focused on healthy adults $[23,27]$, anxious adults [29] or patients in the preoperative period [24,26], and none of them focus on older people. Previous studies investigating the effects of binaural beats on mood of healthy adults used subjective assessment tools and found no significant effects, but none of them used physiological measures [23,27].

The participants reported that the listening to the familiar music with alpha binaural beats was pleasant and helped them relaxed. The nursing staff also reported that 30-minute music listening intervention was suitable and easy to implement for older people. They also reported that the participants appeared relaxed and pleasant when they listened to the music. However, some participants were not used to wear the headphones when listened to music, so it took

\begin{tabular}{|c|c|c|c|c|c|c|}
\hline & \multicolumn{2}{|c|}{ Pre-test } & \multicolumn{2}{|c|}{ Post-test } & \multirow[b]{2}{*}{$z$} & \multirow[b]{2}{*}{$\boldsymbol{P}$} \\
\hline & Mean & SD & Mean & SD & & \\
\hline \multicolumn{7}{|l|}{ 30-minute Immediate effect } \\
\hline Heart rate (beats/min) & 70.11 & 9.3 & 66.69 & 9.48 & -4.075 & $<0.001$ \\
\hline Systolic blood pressure $(\mathrm{mmHg})$ & 128.57 & 16.94 & 119.97 & 14.79 & -5.117 & $<0.001$ \\
\hline Diastolic blood pressure $(\mathrm{mmHg})$ & 71.91 & 13.81 & 71.51 & 12.13 & -0.349 & 0.73 \\
\hline LF norm (nu) & 37.27 & 11.73 & 31.51 & 13.57 & -2.342 & 0.019 \\
\hline HF norm (nu) & 45.81 & 14.86 & 50.5 & 18.01 & -1.974 & 0.048 \\
\hline LF/HF ratio & 0.92 & 0.7 & 0.8 & 0.51 & -1.029 & 0.304 \\
\hline \multicolumn{7}{|l|}{ Five-day effect } \\
\hline Depression score & 10.97 & 1.51 & 7.61 & 2.08 & -4.83 & $\mathrm{P}<0.001$ \\
\hline
\end{tabular}

some time for the staff to explain and teach the participants how to use the headphones.

\section{Study limitations}

There are some limitations to this study that may limit the generalizability of its findings. The sample size was small, and the study was only conducted in a single site. Our study found that familiar music with alpha binaural beats significantly reduced heart rate, systolic blood pressure, and LF norm of HRV. Regarding the statistical significant effect of alpha binaural beats, there is however a possible confounding variable which might affect our conclusion. In our study, each participant listened to the familiar music with alpha binaural beats embedded. It is possible that the reduction of the heart rate, systolic blood pressure, and LF norm of HRV were explained by the effect of the familiar relaxation music itself, not by the presence of the binaural beats. Therefore, an experimental design is needed to compare the effects of familiar music with alpha binaural beats to the same music without the binaural beats. In addition, future studies may examine the long-term effect of music with binaural beats with increased sessions on brainwave activity of older people. More research with larger sample size and use of experimental designs, such as randomized controlled trial, are needed to provide more empirical evidence of the effects of familiar music with alpha binaural beats for elderly people with depressive symptoms.

\section{Conclusion}

As the population of older people increases, the number of older patients with depression is expected to rise. Depression is a serious condition of clinical importance and it can affect functional status and quality of life of older people as well as their caregivers and health care 
resources. With consideration of the adverse effects and high costs of antidepressants used in treating depression of the elderly population, it is important to use inexpensive and viable non-pharmacological interventions along or in combination with medications. Familiar music listening with alpha binaural beats has the potential to decrease heart rate, systolic blood pressure, and HRV, and promote a relaxing and calming state for older people with depressive symptoms, and further reduce their depressive symptoms. Nurses can use this type of music intervention as part of their holistic care approach and build therapeutic relationships with older people with depressive symptoms in long-term care settings. Familiar music with alpha binaural beats can be a noninvasive and suitable intervention to promote relaxation and alleviate negative emotion in older people with depressive symptoms.

\section{Conflict of interest}

None.

\section{Acknowledgements}

This project was supported by National Science Council (NSC98-2314-B-277-001-MY2), Taiwan.

\section{References}

1. Barua A, Ghosh MK, Kar N, et al. Prevalence of depression in the elderly. Ann. Saudi. Med $31(6), 620-624$ (2011).

2. Bryant C, Jackson H, Ames D. Depression and anxiety in medically unwell older adults: prevalence of short-term course. Int Psychogeriatr 21(4), 754-763 (2009).

3. Hsieh MH, Lai TJ. Depression in late life: current issues. Taiwanese J. Psychiatry 19, 85-99 (2005).

4. Lin LC, Wang TG, Chen MY, et al. Depressive symptoms in long-term care residents in Taiwan. J. Adv. Nurs. 51, 30-37 (2005).

5. American Foundation for Suicide Prevention. [Internet]. [Cited 2015 September 23] https://www.afsp.org/understanding-suicide/facts-and-figures (2015)

6. Bottion CMC, Barcelos-Fereira R, Ribeiz SRI. Treatment of Depression in Older Adults. Curr. Psychiatry Rep. 14(4), 289-297 (2012).

7. Trollor JN, Anderson TM, Sachdev PS, et al. Prevalence of mental disorders in the elderly: The Australian national mental health and well-being survey. Am. J. Geriatr. Psychiatry 15(6), 455-466 (2007).

8. Licht-Strunk E, van der Windt DA, et al. The prognosis of depression in older patients in general practice and the community. A systematic review. Fam. Pract 24, 168-180 (2007).

9. Coupland CAC, Dhiman P, Barton G, et al. A study of the safety and harms of antidepressant drugs for older people: a cohort study using a large primary care database. Health. Technol. Assess 15(28), 1-5 (2011).

10. Rapoport M, Mamdani M, Shulman KI, et al. Antipsychotic use in the elderly: shifting trends and increasing costs. Int. J. Geriatr.
Psychiatry 20(8), 749-53 (2005).

11. Lee HP, Su YC, Hou SY. The application of relaxation techniques and biofeedback to an outpatient with depression disorder: a nurse's experience. J. Nurs 56(6), 101-108 (2009).

12. Chen WJ, Chang CF, Chou LS, et al. Effect of cognitive-behavioral group therapy for female outpatients with depressive disorder. Taiwanese J. Psychiatry 22(1), 37-46 (2008).

13. Rizzo M, Creed F, Goldberg D, et al. A systematic review of non-pharmacological treatments for depression in people with chronic physical health problems. J. Psychosom. Res 71, 18-27 (2011).

14. Chen SC, Chiu TH, Hung SY, et al. The effects of group music therapy on depression and quality of life among elders attending community day-care services. J. Long. Term. Care 12(4), 377-389 (2008).

15. Tung HT, Chen KM. Effects of music therapy on cognition, behavior problems, and depression among demented older adults in long-term care facilities. J. Evidence. Based. Nur 3(4), 309-318 (2007).

16. Chen TA, Wu YT, Lee MB, et al. Effects of exercise on depression symptoms, physical function, and quality of life in community-dwelling elderly. Formosan J. phys. Ther 34(3), 209-218 (2009).

17. Snyder M, Chlan L. Music therapy. Annu. Rev. Nurs. Res. 17, 3-25 (1999).

18. Koch ME, Dain ZN, Ayoub C, et al. The sedative and analgesic sparing effect of music. Anesthesiology 89, 300-306 (1998).

19. Taut M. Neuropsychological processes in music perception and their relevance in music therapy. In RF Unkefer (Ed.), Music therapy in the treatment of adults with mental disorders. New York: Schirmer, USA
3-32 (1990).

20. Blood AJ, Zatorre RJ. Intensely pleasurable responses to music correlate with activity in brain regions implicated in reward and emotion. Proc. Natl. Acad. Sci. U S A 98(20), 11818-11823 (2001)

21. Menon V, Levitin DJ. The rewards of music listening: response and physiological connectivity of the mesolimbic system. Neurolmage 28(1), 175-184 (2005).

22. Oster G. Auditory beats in the brain. Sci. Am 229, 94-102 (1973).

23. Wahbeh H, Calabrese C, Zwickey H. Binaural beat technology in humans: a pilot study to assess psychologic and physiologic effects. J. Altern. Complement. Med 13(1), 25-32 (2007).

24. Chuter EE, Allan M, Laws D. A pilot study comparing reduction of anxiety by binaural beat audio and patient-selected music in the pre-operative period. Anaesthesia 62, 310 (2007).

25. Liao MM, Osborn IP. The effect on HemiSync music on intraoperative stress reduction in patients having awake-surgery for Parkinson's disease or dystonia. J. Neurosurg. Anesthesiol 18(4), 330-331 (2006).

26. Padmanabhan R, Hildreth AJ, Laws D. A prospective, randomized, controlled study examining binaural beat audio and pre-operative anxiety in patients undergoing general anaesthesia for day case surgery. Anaesthesia 60, 874-877 (2005).

27. Wahbeh, H, Calabrese C, Zwickey H. Binaural beat technology in humans: a pilot study to assess neuropsychologic, physiologic, and electroencephalographic effects. J. Altern. Complement. Med 13(20), 199-206 (2007).

28. Huang TL, Charyton C. A comprehensive 
Familiar Music Listening with Binaural Beats for Older People with Depressive Symptoms in Retirement Research Homes

review of the psychological effects of brainwave entrainment. Altern. Ther. Health. Med 14(5), 38-50 (2008).

29. Le Scouarnec RP, Poirier R-M, Owens JE, et al. Use of binaural beat tapes for treatment of anxiety: a pilot study of tape preference and outcomes. Altern. Ther. Health. Med 7(1), 58-63

$$
\text { (2001). }
$$

30. Kamath MV, Fallen EL. Power spectral analysis of heart rate variability: A noninvasive signature of cardiac autonomic function. Crit. Rev. Biomed. Eng 21, 245-311 (1993).

31. Sheikh Jl, Yesavage JA. Geriatric depression scale (GDS): present evidence and develop- ment of a shorter version. Clin. Gerontol 5 165-173 (1986)

32. Jongenelis K, Pt AM, Eisses AMH, et al. Diagnostic accuracy of the original 30 -item and shortened versions of the Geriatric Depression Scale in nursing home patients. Int. J. Geriatr. Psychiatry 20, 1067-1074 (2005). 